\title{
QUALITY OF LIFE AND PAIN IN DOGS WITH EARLY-STAGE MAMMARY TUMOURS
}

\author{
Larissa C. FAUSTINO ${ }^{1}$ and Maria Anete LALlO ${ }^{1,2^{*}}$ \\ ${ }^{1}$ Veterinary Medicine, Cruzeiro do Sul University (UNICSUL), São Paulo, Brazil; \\ ${ }^{2}$ Environmental and Experimental Pathology, Paulista University (UNIP), \\ Rua Dr. Bacelar 1212, São Paulo, 04026002, Brazil
}

(Received 22 November 2014; accepted 22 June 2015)

Quality of life (QOL) was evaluated in bitches with mammary tumours (MTs) by applying a scale to assess QOL in dogs with pain secondary to cancer. Two groups were constituted: Group 1 with 80 bitches with MT, oncologically classified as stage I (mean age $\pm \mathrm{SD}=9.9 \pm 3.8$ years), and Group 2 consisting of 80 healthy bitches without MT (mean age: $7.7 \pm 1.8$ years). The results were based on responses from owners using a standardised, internationally accepted pain-scale questionnaire. This prospective and descriptive study showed that $63 \%$ of the dogs in Group 1 had a change in QOL due to the presence of MT. The risk of QOL impairment was 2.1 times higher in Group 1 animals than in bitches without MT. Mammary tumour increased the presence of pain 8.3 times and defecation difficulties 10 times. It can be concluded that even small MTs can elicit pain in dogs, which interferes with their quality of life.

Key words: Cancer pain, dog, mammary tumour, pain, quality of life

Mammary tumours (MTs) are the most common type of tumour in intact female dogs. The clinical presentation of canine MTs is variable; they occur either as single or multiple nodules, ranging from well-circumscribed nodules with stationary growth to large and sometimes ulcerated nodules which grow rapidly and become fixed to adjacent tissues or display other signs of malignancy (Lana et al., 2007). Early surgical removal is recommended for canine MT (Lana et al., 2007), but the veterinary practice indicates that because of the apparent absence of pain or the lack of sufficient commitment, many owners and even veterinarians postpone surgical treatment of MT, thus compromising disease outcomes and the patients' quality of life (QOL).

The difficulty in assessing pain in animals has been a limiting factor to their identification and treatment. For this reason, the American College of Veterinary Anesthesiologists (ACVA) established that a stimulus considered painful in human beings should also be considered painful in animals and the inability to

*Corresponding author; E-mail: anetelallo@hotmail.com; Phone: 0055 (11) 999869607; Fax: 0055 (11) 55864000 
verbalise at no time should be interpreted as absence of pain sensation (ACVA, 1998). The availability of quantitative measures of chronic pain that are valid and reliable in clinical patients is crucial for the development and testing of interventions (e.g. drugs or surgical procedures) designed to reduce such pain. A detailed behaviour-based assessment of chronic pain performed by the owner is routinely relied on when making clinical decisions. This offers the advantage of an extended assessment of a dog in its typical environment by someone who is most knowledgeable about its behaviour. Although an owner's detailed assessment can be quite useful, few programmes have reported the development of an owner-completed questionnaire for use as an outcome assessment tool in clinical studies. The impact of pain associated with chronic diseases on QOL has been evaluated in dogs (Wiseman-Orr et al., 2004, 2006). In this study, we used the QOL assessment questionnaire developed by Yazbek and Fantoni (2005) because it is a questionnaire validated for pain secondary to cancer.

In humans, cancer pain affects $50 \%$ of patients throughout the course of the disease. When evaluating only patients in the advanced stage, the incidence of pain can reach $75 \%$ (Sakata, 2004). In many types of canine cancer, however, the presence of pain has not been assessed. In cancer, pain may result from a variety of causes: it may be caused by the tumour itself, by metastasis or by paraneoplastic syndromes, and it can be the result of chemotherapy and radiotherapy (Lester and Gaynor, 2000; Teixeira, 2003). Quality of life assessments are considered to be among the principal endpoints in human clinical trials (Freeman et al., 2005) and are now commonplace in primary care practice (Mellanby et al., 2003). Only in the recent decade has QOL been extensively studied and measured in companion animal medicine (Brown et al., 2008). Few studies reveal the true associations between tumours observed in humans and dogs, because assessment of the QOL life in pets is rarely used in clinical studies. There is a consensus that QOL in pets should be more broadly defined as states of comfort or discomfort representing a combination of physical and non-physical factors (McMillan, 2000; Mellanby et al., 2003). For this reason, the aim of this study was to evaluate whether the presence of mammary nodules can affect the QOL of dogs, and thus to estimate the degree of morbidity in such cases.

\section{Materials and methods}

Two groups were constituted: Group 1 (G1-MT) comprising 80 bitches with MT and Group 2 (G2-C) consisting of 80 bitches without MT. Bitches of Group G1-MT had only one non-ulcerated mammary tumour $1-3 \mathrm{~cm}$ in diameter, and according to the World Health Organization Clinical Staging System (the TNM system) all animals were classified as $\mathrm{T} 1(<3 \mathrm{~cm}$ maximum diameter), N0 (lymph nodes not compromised by metastases) and M0 (no distant metastasis) 
(Philbert et al., 2003). Exclusion criteria for this group were tumours larger than $4 \mathrm{~cm}$, ulcerated, recurrent tumours, animals undergoing chemotherapy or surgery, presence of other tumours, and other concomitant diseases. The age of these dogs ranged from 5 to 19 years (mean age $\pm \mathrm{SD}=9.9 \pm 3.8$ years) and the body weight median was $15.4 \pm 10.2 \mathrm{~kg}$. The breeds represented in this group were crossbreed dogs $(n=29)$, standard Poodle $(n=17)$, Rottweiler $(n=11)$, Pit Bull $(\mathrm{n}=8)$, Labrador $(\mathrm{n}=7)$, Boxer $(\mathrm{n}=2)$, Pinscher $(\mathrm{n}=2)$, German Shepherd $\operatorname{Dog}(n=2)$, Bull Terrier $(n=1)$, and Cocker Spaniel $(n=1)$. The diagnosis of mammary neoplasm was done by histopathological examination of excisional biopsy samples after surgical treatment (Misdorp et al., 1999).

Group G2-C group consisted of healthy, non-neutered bitches without MT, aged 5 to 16 years (mean $7.7 \pm 1.8$ years) and with a body weight median of $13.5 \pm$ $11.9 \mathrm{~kg}$. The major breeds belonging to Group 2 were crossbreeds $(\mathrm{n}=26)$, standard Poodle $(\mathrm{n}=14)$, Pinscher $(\mathrm{n}=12)$, Labrador $(\mathrm{n}=8)$, Pit Bull $(\mathrm{n}=5)$, Dachshund ( $(n=5)$, Maltese $(n=3)$, Akita $(n=2)$, Rottweiler $(n=2)$, German Shepherd Dog $(n=2)$, and Chow Chow $(n=1)$. The exclusion criteria for this group were the presence of diseases including any tumours, and the dogs were not being subjected to any kind of treatment.

We used the 'Scale to assess quality of life in dogs with pain secondary to cancer' validated by Yazbek and Fantoni (2005), which consists of a questionnaire of twelve questions and four possible response alternatives. Each question is worth 0 (zero) to 3 (three) points, with a maximum total score of 36 points; zero is considered the worst quality of life and 36 points the best quality. The questions cover information about general behaviour, interaction with owners, and assessment of pain, appetite, fatigue, sleep disturbances, stomach and intestinal problems, defecation, and urination.

The $\chi^{2}$-test was used to compare the prevalence of factors analysed by the survey, and differences were considered significant at $\mathrm{P}<0.05$. The results of the questionnaire allowed us to calculate the relative risks from the prevalences of all categories. All statistical analyses were performed using standard software (SPSS 15.0 for Windows, SPSS, Chicago, Illinois, USA).

\section{Results}

We found that $15 \%$ of the animals had a score 0 , which is considered a totally changed QOL, and $48.75 \%$ obtained temperament scores 1 and 2 which indicate some episodes of alteration and slightly altered temperament, respectively. Thus we showed that $63.75 \%$ of bitches with MT had changes consistent with decreased QOL, and only $36.25 \%$ of the animals with MT had normal QOL.

The owner questionnaire aimed at assessing QOL and pain in dogs with MT revealed that the risk of QOL impairment was 2.1 times higher in bitches 
with MT than in those without MT. We observed that the risk of cancer patients developing changes in temperament (prevalence of increased aggression, isolation and irritability in the MT group: 50\%) increased 2.6 times as compared to the control group (prevalence of increased aggression, isolation and irritability: $10 \%$ ). Owners of dogs with MT were 8.3 times more likely to report pain than those of bitches without MT. Thus, pain was one of the predominant reasons for the occurrence of behavioural changes, and it can explain many other changes visibly present in bitches with MT. The presence of changes in defecation (difficult defecation, dyschezia) was 10 times more frequent in the MT group. This sign is often related to the presence of pain, and its frequency corroborates the occurrence of pain in patients with MT. This study demonstrated that the risk of vomiting episodes increased 3.7 times and the presence of intestinal disorders 1.8 times in Group MT. Bitches with MT were attended to by their owners approximately 21 times more than bitches without MT, which fact reveals an insecurity due to pain or discomfort caused by the presence of tumours in dogs with MT (Table 1).

\section{Table 1}

Prevalence and risk analysis based on the results obtained through the pain and quality of life questionnaire for canine mammary tumour patients

\begin{tabular}{lccc}
\hline Factor & $\begin{array}{c}\text { Group 1- } \\
\text { dogs without MT } \\
(\%)\end{array}$ & $\begin{array}{c}\text { Group 2- } \\
\text { dogs with MT } \\
(\%)\end{array}$ & $\begin{array}{c}\text { Relative } \\
\text { risk }\end{array}$ \\
\hline - Disturbing the animal's life & $18 / 50(36)$ & $62 / 80(77.50)^{*}$ & $2.10^{*}$ \\
2 - Does not keep doing the same things & $27 / 50(54)$ & $46 / 80(57.50)^{*}$ & $1.0^{*}$ \\
3 - Loses hygiene habits & $12 / 50(24)$ & $51 / 80(63.75)^{*}$ & $5.0^{*}$ \\
4 - Behavioural changes & $5 / 50(10)$ & $40 / 80(50.00)^{*}$ & $2.6^{*}$ \\
5 - Presence of pain & $5 / 50(10)$ & $67 / 80(83.75)^{*}$ & $8.3^{*}$ \\
6 - Change of appetite & $40 / 50(80)$ & $41 / 80(51.25)^{*}$ & $0.6^{*}$ \\
7 - Displays tiredness & $16 / 50(32)$ & $54 / 80(67.50)^{*}$ & $2.0^{*}$ \\
8 - Shows changes in sleep & $14 / 50(28)$ & $38 / 80(47.50)^{*}$ & $1.6^{*}$ \\
9 - Displays respiratory distress or vomiting & $5 / 50(10)$ & $30 / 80(37.50)^{*}$ & $3.7^{*}$ \\
10 - Presents intestinal disorders & $15 / 50(30)$ & $44 / 80(55.00)^{*}$ & $1.8^{*}$ \\
11 - Presence of postural changes to defecate & $2 / 50(4)$ & $33 / 80(41.20)^{*}$ & $10.2^{*}$ \\
12 - Increase of grace / family care & $1 / 50(2)$ & $34 / 80(42.50)^{*}$ & $21.0^{*}$ \\
\hline
\end{tabular}

${ }^{*}$ Results of $\chi^{2}$-test for two independent proportions, $\mathrm{P}<0.05$

In Group G1-MT, we observed that $65 \%(52 / 80)$ of mammary tumours were malignant and $35 \%(28 / 80)$ were benign. Adenomas (17/28) and fibroadenomas $(11 / 28)$ were the most prevalent benign mammary tumours. Histological analysis demonstrated that 52 dogs had carcinoma, including tubular carcinoma (27/52), papillary carcinoma (5/52), solid carcinoma (15/52), and complex carcinoma $(5 / 52)$. 


\section{Discussion}

The assessment of pain in animals, while often difficult, is extremely important. It is likely that the tolerance of pain by an individual animal varies greatly, and this is further complicated by the innate ability of dogs to mask significant disease and pain. The most important people in the assessment process are the owners. The veterinarian must work closely with the owner to capture this information. Importantly, the veterinarian needs to capture information about behaviours or activities that have changed as a result of the cancer. Tumour characteristics that decisively contribute to the occurrence of pain are tumour stage, size, presence of tumour ulceration, recurrence, and metastases. However, little is known about the pain caused by small, isolated mammary tumours in bitches, which was the main motivation behind this study.

In the clinical practice, the presence of small mammary nodes in dogs cannot demand much attention from the owner and the veterinarian because these tumours are of small size and the patient shows few clinical signs. Thus, wrongly, it is believed that the presence of little lumps does not interfere with the QOL of dogs. However, this study showed that about $63.75 \%$ of dogs with earlystage MT had a change in their QOL, and the risk of QOL changes increased 2.1 times in dogs with MT.

The behavioural changes (aggression, difficulty in defecation) and the QOL of dogs were related to the presence of pain, because animals with MT had a 8.3 times higher risk of pain. The most common sign of pain is a change in behaviour, such as changes in personality or attitude, especially vocalisation when the painful region is touched, self-mutilation, changes in fur appearance and hygiene, as well as changes in posture and gait (Loney, 2010), which is consistent with what we found in this study. Many of these changes are manifested in subtle form and are barely noticeable during the daily routine of the affected animal. These small alterations in the animal's behaviour are observed only by the owners who report them when questioned on the subject. Thus, it is clear that tumour pain is not only present in dogs with large or ulcerated tumours but also in those with tumours of smaller size. In this case, defensive aggression (Horwitz and Neilson, 2008) is probably the most typical aggressive behavioural pattern.

We found that the risk of vomiting episodes increased 3.7 times and the presence of intestinal disorders 1.8 times in dogs with MT. Gastrointestinal disturbances are the main adverse effects reported in animals subjected to chemotherapy (Rassnick et al., 2007). In this study, no patient received any kind of treatment or chemotherapy. These signs are probably related to the presence of tumours or metastases. Horwitz and Neilson (2008) argue that excessive licking can also be considered a sign that the animal is seeking the attention of its owners or experiences pain, itching, or anxiety disorders. Furthermore, excessive in- 
take of hairs or secretions from licking the tumour can cause gastrointestinal discomfort (Duque and Valadão, 2008; Gaynor, 2008).

According to the American College of Veterinary Anesthesiologists (ACVA, 1998), the pain and suffering of animals are clinically important conditions that affect the quality of life in the short and long term. Besides the ethical considerations of pain suppression, analgesic usage has many other proven benefits: it reduces emotional stress, inhibits the release harmful substances into the body, facilitates patient recovery, and reduces mortality and morbidity (Loney, 2010). Advanced cancer is known to be responsible for many pathophysiological changes related to pain and reduced QOL. The results of this study show that the presence of pain impairs QOL in bitches with mammary tumours of different sizes, including small ones. Thus, veterinarians must be alert for the treatment of pain, even in patients that apparently do not exhibit very clear clinical signs of pain.

We conclude that mammary tumours decrease the quality of life in bitches, which is reflected in changes associated with pain including postural changes for defecation or urination, loss of hygiene habits, breathing difficulty, behavioural changes, and increased attention demand from the owner due to a feeling of insecurity.

\section{Acknowledgement}

We thank the veterinary clinics and hospitals that participated in this study.

\section{References}

ACVA (American College of Veterinary Anesthesiologists) (1998): Position paper on the treatment of pain in animals. J. Am. Vet. Med. Assoc. 213, 628-630.

Brown, D. C., Boston, R. C., Coyne, J. C. and Farrar, J. T. (2008): Ability of the canine brief pain inventory to detect response to treatment in dogs with osteoarthritis. J. Am. Vet. Med. Assoc. 233, 1278-1283.

Duque, J. C. M. and Valadão, C. A. A. (2008): Pain management in cancer patients. In: Daleck, C. R., De Nardi, A. B. and Rodasky, S. (eds) Oncology in Dogs and Cats [in Portuguese]. Roca, São Paulo. pp. 228-247.

Freeman, L. M., Rush, J. E., Farabaugh, A. E. and Must, A. (2005): Development and evaluation of a questionnaire for assessing health-related quality of life in dogs with cardiac disease. J. Am. Vet. Med. Assoc. 226, 1864-1868.

Gaynor, J. S. (2008): Control of cancer pain in veterinary patients. Vet. Clin. North Am. Small Anim. Pract. 38, 1429-1448.

Horwitz, D. F. and Neilson, J. C. (eds) (2008): Canine and Feline Behaviour [in Portuguese]. Artmed, Porto Alegre. $662 \mathrm{pp}$.

Lana, S. E., Rutteman, G. R. and Withrow, S. J. (2007): Tumors of the mammary gland. In: Withrow, S. J. and Vail, D. M. (eds) Withrow \& MacEwen's Small Animal Clinical Oncology. 4th edition. Saunders Elsevier, St. Louis. pp. 619-633. 
Lester, P. and Gaynor, J. S. (2000): Management of cancer pain. Vet. Clin. North Am. Small. Anim. Pract. 30, 951-966.

Loney, A. (2010): Oncology pain in veterinary patients. Top. Companion. Anim. Med. 25, 32-44.

McMillan, F. D. (2000): Quality of life in animals. J. Am. Vet. Med. Assoc. 216, 1904-1910.

Mellanby, R. J., Herrtage, M. E. and Dobson, J. M. (2003): Owners assessments of their dog's quality of life during palliative chemotherapy for lymphoma. J. Small Anim. Pract. 44, $100-103$.

Misdorp, W., Else, R. W., Hellmén, E. and Lipscomb, T. P. (1999): Histological classification of mammary tumors of the dog and the cat. Armed Forces Institute of Pathology, the American Registry of Pathology and The World Health Organization Collaborating Center for Worldwide Reference on Comparative Oncology, Washington, D.C. pp. 11-29.

Philbert, J. C., Snyder, P. W., Glikman, N., Knapp, D. W. and Waters, D. J. (2003): Influence of host factors on survival in dogs with malignant mammary gland tumors. J. Vet. Intern. Med. 17, 102-106.

Rassnick, K. M., McEntee, M. C., Erb, H. N., Burke, B. P., Balkman, C. E., Flory, A. B., Kiselow, M. A., Autio, K. and Gieger, T. L. (2007): Comparison of 3 protocols for treatment after induction of remission in dogs with lymphoma. J. Vet. Int. Med. 21, 1364-1373.

Sakata, R. K. (2004): Cancer pain. In: Sakata, R. K. and Issy, A. M. (eds) Outpatient Medical and Hospital Guides, UNIFESP - Pain [in Portuguese]. Manole, Barueri. pp. 117-125.

Teixeira, M. J. (2003): Pain in the patient with cancer. In: Teixeira, M. J. (ed.) Pain in the Interdisciplinary Context [in Portuguese]. Maio, Curitiba. pp. 327-341.

Wiseman-Orr, M. L., Nolan, A. M., Reid, J. and Scott, E. M. (2004): Development of a questionnaire to measure the effects of chronic pain on health-related quality of life in dogs. Am. J. Vet. Res. 65, 1077-1084.

Wiseman-Orr, M. L., Scott, E. M., Reid, J. and Nolan, A. M. (2006): Validation of a structured questionnaire as an instrument to measure chronic pain in dogs on the basis of effects on health-related quality of life. Am. J. Vet. Res. 67, 1826-1836.

Yazbek, K. V. B. and Fantoni, D. T. (2005): Validity of a health-related quality-of-life scale for dogs with signs of pain secondary to cancer. J. Am. Vet. Med. Assoc. 226, 1354-1358. 